\title{
CARACTERÍSTICAS SALIVALES RELACIONADAS CON CARIES Y CÁLCULO DENTAL EN PERSONAS MAYORES DE 50 AÑOS
}

\author{
${ }^{1}$ Carmen Alodia Martínez López, ${ }^{2}$ Tatiana Marcela Agudelo Osorio, ${ }^{2}$ Ana Lucía Espejo Pérez, ${ }^{2}$ Zareth Garzón Ramírez, \\ ${ }^{2}$ Katherine Martínez Maldonado. \\ ${ }^{1}$ Odontóloga U. Santo Tomás, Especialista en Ortopedia Maxilar U. Antonio Nariño, Docente U. Santo Tomás (Colombia). \\ ${ }^{2}$ Estudiante X semestre F. de Odontología U. Santo Tomás, Bucaramanga (Colombia).
}

Autor responsable de correspondencia: Carmen Alodia Martínez López

Correo electrónico: carmenmartinez82@yahoo.com

\begin{abstract}
RESUMEN
Objetivo: Relacionar las características salivales con presencia de caries y cálculos dentales en pacientes adultos mayores que asisten a las Clínicas Odontológicas de la Universidad Santo Tomás en Bucaramanga.

Materiales y métodos: Se realizó un estudio de corte transversal en una población de 46 personas mayores de 50 años. Las variables dependientes utilizadas fueron $\mathrm{pH}$, aclaramiento y flujo salival, las independientes fueron las sociodemográficas, caries coronal, caries radicular, cálculo dental y hábitos de higiene. Los datos fueron tabulados y analizados estadísticamente con el Test Chi y el exacto de Fisher para variables cualitativas y para variables cuantitativas $t$ Student.

Resultados: La prevalencia de caries coronal fue del $65,2 \%$ y de caries radicular fue del $34,8 \%$. El consumo de medicamentos es un posible factor de riesgo para el aumento de $\mathrm{pH}$ salival. El 84,8\% de la población tienen tiempo de aclaramiento mayor de 15 minutos. El flujo salival elevado se considera un factor de riesgo para el pH salival básico (RP 1,54) y para presencia de cálculo dental. Un porcentaje considerable de mujeres (51,7\%) presentó flujo salival estimulado disminuido mientras que apenas el 11,8\% de los hombres reportó esta característica.

Conclusiones: La tasa de flujo salival estimulado disminuido prevalece más en las mujeres que en los hombres; tener 56 años o más, aumenta el riesgo de disminución en flujo salival estimulado, y éste se relaciona directamente con mayor presencia de cálculo dental que resulta ser un condicionante de acúmulo de placa que provoca caries radicular en los adultos mayores. [Martínez CA, Agudelo TM, Espejo AL, Garzón Z, Martínez K. Características salivales relacionadas con caries y cálculo dental en personas mayores de 50 años. Ustasalud 2011; 10: 43 - 53]
\end{abstract}

Palabras clave: Caries dental, Higiene oral, Saliva, Cálculo dental.

\section{SALIVARY CHARACTERISTICS RELATED TO DENTAL CARIES AND DENTAL CALCULLUS IN PEOPLE OVER 50 YEARS}

\begin{abstract}
Objective: To relate the characteristics and presence of dental caries and salivary calculus in elderly patients that attended the Dental Clinics at Santo Tomas University in Bucaramanga.

Methods: A cross sectional study was done with 46 people over 50 years. The dependent variables used were pH, clearance and salivary flow, the independent variables were sociodemographic, coronal caries, root caries, dental calculus and dental hygiene. Data were tabulated and analyzed statistically using $\mathrm{Chi}^{2}$ test and Fisher exact test for qualitative variables and Student $t$ test for quantitative variables.

Results: The prevalence of coronal caries was $65.2 \%$ and root caries was $34.8 \%$. The consumption of drugs is a possible risk factor for the increase in salivary pH. $84.8 \%$ of the population has higher clearance time of 15 minutes. High salivary flow is considered a risk factor for salivary pH basic (OR: 1.54) and presence of dental calculus. A considerable percentage of female (51.7\%) had decreased stimulated salivary flow while only $11.8 \%$ of men reported this feature.

Conclusions: The stimulated salivary flow rate decreased more prevalent in women than in men, being 56 years or more increases the risk of decline in stimulated salivary flow, and this is directly related to increased presence of dental calculus turns out to be a determinant of plaque buildup leading to root caries in older adults.
\end{abstract}

Key words: Dental caries, Oral hygiene, Saliva, Dental calculus.

Recibido para publicación: 10 de mayo de 2011. Aceptado para publicación: 10 de junio de 2011. 


\section{INTRODUCCIÓN}

La Organización Panamericana de la Salud (OPS) define el estado de salud entre los envejecidos no en términos de déficit sino de mantenimiento de la capacidad funcional (OPS/OMS, 1982). Las alteraciones en saliva respecto a los cambios del pH, flujo, capacidad buffer y aclaramiento de azúcares pueden aumentar la susceptibilidad para la aparición de enfermedades bucales tales como candidiasis, caries y periodontopatías. ${ }^{1,2}$ La saliva, sustancia secretada por las glándulas salivales, transparente, de viscosidad variable, compuesta por agua, proteínas y sales minerales es necesaria para la función normal de los sistemas de defensa de la cavidad oral, ${ }^{3}$ dentro de estos se cuentan: aclaramiento de azúcares, remineralización dental, modulación de concentración de microorganismos, participación del proceso digestivo, ${ }^{4}$ mantenimiento del $\mathrm{pH}$ y, en consecuencia, de la integridad de los tejidos dentales. ${ }^{5}$ Posee propiedades como la alta viscosidad, baja solubilidad, elasticidad y adhesividad, ${ }^{6}$ secreción entre 500$700 \mathrm{ml}$ por día en un adulto, con un volumen medio en la boca de $1 \mathrm{ml} .{ }^{7}$ La cuales son esenciales para que la salud bucal se mantenga. ${ }^{8}$ Una disminución en la tasa de flujo salival conduce a alteraciones de $\mathrm{pH}$, reportándose bajas considerables en pacientes a medida que la secreción salival decrece. ${ }^{9}$ Cualquier factor que ocasione un incremento del $\mathrm{pH}$, puede iniciar la formación de cálculos, puesto que el flujo salival aumentado ocasiona un ambiente bucal alcalino. ${ }^{10}$ Los rangos normales de $\mathrm{pH}$ salival oscilan entre 5.7 y 7.6 , el pH de la saliva está próximo a la neutralidad. ${ }^{9}$

Entre las causas más comunes en la reducción salival se encuentran: la ingesta de medicamentos, alteraciones sistémicas crónicas, tratamiento de cáncer de cabeza y cuello que incluya radioterapia o quimioterapia. ${ }^{8,10}$ Autores como Almstahl y colaboradores (2003) han demostrado que los individuos que han padecido disminución en el flujo salival y un $\mathrm{pH}$ ácido desarrollan caries coronal y radicular especialmente en adultos mayores. ${ }^{11}$ Otra característica importante de la saliva es el tiempo de aclaramiento salival, que se ve afectado por la tasa del flujo salival estimulado. De manera que el aclaramiento salival en las diferentes zonas de la boca presenta una eliminación diferente, ya que entre más cerca se encuentre al drenaje de los ductos de las glándulas salivales, su eliminación es más rápida, debido a que la velocidad de la saliva es más rápida en estas zonas. ${ }^{6,7,12}$

El examen odontológico en la clínica de adulto mayor contempla flujo salival. El propósito de este estudio fue relacionar $\mathrm{pH}$, flujo salival y aclaramiento con presencia de caries y cálculo dental en pa- cientes mayores de 50 años atendidos en las clínicas odontológicas de la USTA, con la intención de justificar el anexo en la historia de las características salivales $\mathrm{pH}$ y aclaramiento.

\section{MATERIALES Y MÉTODOS}

Se realizó un estudio de corte transversal. La población de estudio estuvo constituida por pacientes de ambos géneros que asistieron a la consulta odontológica en las Clínicas Odontológicas de la Universidad Santo Tomás durante el segundo semestre de 2010. Los criterios selección fueron: pacientes mayores de 50 años activos en el segundo semestre de 2010 con historia clínica aprobada, que aceptaran participar en la investigación, con ubicación telefónica comprobada, presencia de más de un diente en boca, ausencia de patología de glándulas salivales y que no se encontraran bajo tratamiento de radioterapia de cabeza y cuello. Del universo conformado por 294 personas cumplieron los criterios de inclusión 46 sujetos que constituyeron la población de estudio.

Para realizar el trabajo se acató la normatividad establecida en la Resolución No. 008430 de 1993 del Ministerio de Protección Social, por lo cual se garantizó el beneficio, la privacidad y confidencialidad de la información y autonomía.

Se efectuó una estandarización de los procesos previa a la realización de la prueba piloto que se realizó con siete pacientes. El procedimiento consistió en la explicación verbal del proyecto, se resolvieron inquietudes y se firmó el consentimiento informado. Se consignaron en el instrumento los datos correspondientes a la anamnesis (características sociodemográficas, antecedentes médicos y hábitos de higiene). Se realizó el examen clínico bucal por parte del evaluador y en el laboratorio de Ciencias Básicas se realizó la recolección de las muestras de saliva. Para este proceso el paciente debió enjuagarse la boca y esperar 1 minuto antes de iniciar.

Para realizar la prueba de flujo salival, en reposo, se le solicitó al paciente sentarse en posición cómoda, apoyar la punta de la lengua contra las superficies palatinas de los dientes superiores y evitar movimientos de las mejillas y la mandíbula. Además, se le pidió cerrar los dientes con los labios ligeramente abiertos en posición relajada y dejar caer la saliva por goteo en una jeringa desechable durante tres minutos. Para la recolección de saliva estimulada se entregó una esfera de parafina de $7 \mathrm{~mm}$ de diámetro, se solicitó que la introdujera en boca y que realizara movimientos con ella durante 5 minutos escupiendo en una jeringa desechable.

Para efectuar la prueba de $\mathrm{pH}$ se recogió una gota de saliva estimulada y se llevó a una lámina portaobjetos donde se impregnó con una tira universal de $\mathrm{pH}$. Se esperó 5 minutos, los resultados se obtu- 
vieron de la comparación del color de la tira con la guía de colores.

Para la prueba de aclaramiento de azúcares se utilizó el método de amilasa salival al utilizar la muestra de la saliva estimulada y de no estimulada $(1 \mathrm{ml}$ - $2 \mathrm{ml}$ ), se llevaron 10 gotas de cada muestra a los tubos de ensayo y se agregaron 10 gotas de almidón al $1 \%$ a cada recipiente y 2 gotas $2 \mathrm{M}$ de HCL; los tubos se calentaron a temperatura corporal aproximada (manteniéndolos en las manos durante 15 minutos), y se agregó 1 gota de yodo de cada tubo. Los resultados se registraron en el formato correspondiente.

La base de datos se realizó en Microsoft Excel 2007. Se exportó la información al programa estadístico Stata 11,0 para realizar los respectivos análisis. Para el análisis univariado se calcularon medidas de resumen según la naturaleza de las variables: para las cuantitativas se obtuvieron medidas de tendencia central y dispersión y para las cualitativas frecuencias y porcentajes. En el análisis bivariado se aplicaron las pruebas estadísticas $\mathrm{Chi}^{2}$ para comparar variables cualitativas y $t$ de Student para las variables cuantitativas. Igualmente, se calcularon razones de prevalencia (RP) y sus respectivos intervalos de confianza al 95\% (IC 95\%).

En el análisis multivariado se realizó una regresión robusta de Poisson para calcular las RP ajustadas. Se tomaron como variables de salida flujo salival no estimulado y estimulado disminuido, $\mathrm{pH}$, caries dental y cálculo dental; y como variables explicatorias las sociodemográficas, higiene oral y tasa de flujo salival no estimulado y estimulado tanto disminuido como aumentado, $\mathrm{pH}$ y aclaramiento salival. Se consideró un nivel de significancia de 0,05.

\section{RESULTADOS}

La muestra estuvo conformada por 46 adultos, 29 $(63,0 \%)$ de las cuales eran mujeres. El $43,5 \%$ tenían entre 50 y 55 años, el 43,5\% entre 56 y 70 años y el restante $13 \%$ era mayor de 70 años. El nivel educativo predominante fue la educación básica secundaria con un $39,1 \%$, en cuanto al estrato socioeconómico, la mayoría pertenecía a los estratos tres y cuatro $(67,4 \%)$. Se presentó una prevalencia de caries coronal de $65,2 \%$, caries radicular de $34,8 \%$ y cálculo dental de $76,1 \%$. La prevalencia de caries dental y cálculo fue mayor en hombres, no se hallaron diferencias estadísticamente significativas (Tabla 1).

El valor de $\mathrm{pH}$ salival reportado fue neutro en el $43,5 \%$ y básico en el $56,5 \%$ de los participantes; la tasa de flujo salival no estimulado se encontró disminuida en el 58,7\%, normal en el $28,3 \%$ y aumentada en el 13\% de los participantes. En contraste, la tasa de flujo salival estimulado mostró un compor- tamiento estadísticamente diferente entre el sexo femenino y masculino, mientras más de la mitad de las mujeres presentaba una tasa de flujo salival estimulado disminuida (51,7\%), más del $50 \%$ de los varones presentó una tasa normal $(58,8 \%)$. En relación con el aclaramiento de azúcar en saliva a los 15 minutos, se encontró predominio de la coloración azul, lo cual significa que no hubo degradación de almidón en el $84,8 \%$ de la población. Se observó caries coronal con predominio en el sexo masculino $(70,6 \%)$, en el grupo de edad entre 50 a 55 años $(80,0 \%)$. En cuanto a la caries radicular, se encontró que una mayor prevalencia en el sexo masculino $(41,2 \%)$, en personas de 56 años o más $(42,3 \%)$ y personas con menor educación, analfabeta/primaria, $(50,0 \%)$. Hubo una alta prevalencia de cálculo en ambos sexos siendo de $75,9 \%$ para mujeres y el $76,5 \%$ para los hombres (Tabla 1).

El tener más de 50 años es un posible factor de riesgo para cálculo dental $(\mathrm{RP}=1,02)$. Sin embargo, ningún resultado fue estadísticamente significativo. Del total de personas que consumían medicamentos, el $70,4 \%$ presentaron caries coronal y se observó también como factor de riesgo para cálculo dental.

Los hábitos de higiene oral asociados a la caries coronal mostraron que cepillarse los dientes tres o más veces mantenían sanas las superficies dentales (RP 0,66 IC 95\% 0,45 - 0,95) por lo que se consideró como un factor protector. Visitar al odontólogo una vez al año (RP 0,26 IC 95\% 0,12-0,62 p=0,008) y cuando duele (RP 0,40 IC 95\% 0,23-0,68 p=0,028) son factores protectores para caries radicular y formación de cálculo, estos datos son estadísticamente significativos. Se observó también que hay menor prevalencia de caries radicular en personas que usan diariamente la seda dental $(22,2 \%)$. Con relación a los hábitos de higiene oral asociados a calculo dental se observó que cepillarse tres veces al día (RP 0,74 IC 95\% 0,56 - 0,97 p=0,078) acompañado del uso de seda dental diariamente (RP 0,70 IC 95\% $0,46-1,05 p=0,125)$ son factores protectores para el cálculo dental pero no fueron estadísticamente significativos $(p>0,05)$.

$\mathrm{Al}$ relacionar los factores asociados a la tasa de flujo salival no estimulado disminuida, se encontró una prevalencia menor de flujo salival disminuido en quienes se cepillaban los dientes tres o más veces al día $(0,23-0,75)$ y presentan cálculo localizado $(0,30-0,85)$ comparado con quienes se cepillan los dientes dos veces al día y no presentan cálculos respectivamente. Por otro lado, la prevalencia de flujo salival disminuido fue mayor en quienes usaban enjuague bucal $(1,04-3,35)$ comparado con quienes 
no usaban enjuague. La prevalencia de flujo salival estimulado disminuido fue menor en los participantes de sexo masculino comparado con los de sexo femenino $(0,04$ - 0,96) (Tabla 2, 3 y 4).

Con respecto al hábito de fumar, se encontró que hay mayor prevalencia de $\mathrm{pH}$ básico en las personas que no fuman $(61,5 \%)$, pero no fue estadísticamente significativo. La presencia de cálculo dental asocia- da al pH básico puede concluir que tener cálculos es un factor protector para tener $\mathrm{pH}$ básico, pero no es un dato confiable pues no es estadísticamente significativo (IC 95\% 0,43 - 1,13). El aumento del flujo salival tanto estimulado como no estimulado se consideró un factor de riesgo para $\mathrm{pH}$ salival básico (RP 1,54), aunque no presentó asociación estadísticamente significativa (Tabla 5).

Tabla 1. Características sociodemográficas, propiedades de la saliva, presencia de caries y calculo dental.

\begin{tabular}{|c|c|c|c|c|}
\hline Variables & $\begin{array}{c}\text { Global } \\
46(100)\end{array}$ & $\begin{array}{c}\text { Femenino } \\
29(63,0)\end{array}$ & $\begin{array}{c}\text { Masculino } \\
17(37,0)\end{array}$ & $\mathbf{P}$ \\
\hline \multicolumn{5}{|c|}{ Características sociodemográficas n (\%) } \\
\hline \multicolumn{5}{|l|}{ Edad } \\
\hline $50-55$ & $20(43,5)$ & $13(44,8)$ & $7(41,2)$ & \\
\hline 56- 70 & $20(43,5)$ & $12(41,4)$ & $8(47,1)$ & \\
\hline$>70$ & $6(13,0)$ & $4(13,8)$ & $2(11,8)$ & \\
\hline \multicolumn{5}{|l|}{ Nivel Educativo } \\
\hline Analfabeta & $3(6,5)$ & $2(6,9)$ & $1(5,9)$ & \\
\hline Primaria & $13(28,3)$ & $8(27,6)$ & $5(29,4)$ & \\
\hline Secundaria & $18(39,1)$ & $12(41,4)$ & $6(35,3)$ & \\
\hline Estudios superiores & $12(26,1)$ & $7(24,1)$ & $5(29,4)$ & \\
\hline \multicolumn{5}{|l|}{ Estrato socioeconómico } \\
\hline 1 у 2 & $15(32,6)$ & $8(27,6)$ & $7(41,2)$ & \\
\hline 3 y 4 & $31(67,4)$ & $21(72,4)$ & $10(58,8)$ & \\
\hline \multicolumn{5}{|l|}{ Propiedades de la saliva $n(\%)$} \\
\hline \multicolumn{5}{|l|}{ Valor de pH salival reportado } \\
\hline$<5.7$ (acido) & $0(0,0)$ & $0(0,0)$ & $0(0,0)$ & \multirow{3}{*}{$0,765^{* *}$} \\
\hline 5.7- 7.6 (neutro) & $20(43,5)$ & $12(41,4)$ & $8(47,1)$ & \\
\hline 7.6 ó más (básico) & $26(56,5)$ & $17(58,61)$ & $9(52,9)$ & \\
\hline \multicolumn{5}{|c|}{ Tasa de flujo salival no estimulado } \\
\hline $0-0.25 \mathrm{ml} / \mathrm{min}$ (disminuido) & $27(58,7)$ & $18(62,1)$ & $9(52,9)$ & \multirow{3}{*}{$0,309^{\prime}$} \\
\hline $0.25-0.35 \mathrm{ml} / \mathrm{min}$ (normal). & $13(28,3)$ & $9(31,0)$ & $4(23,5)$ & \\
\hline$>0.35 \mathrm{ml} / \mathrm{min}$. (aumentado) & $6(13,0)$ & $2(6,9)$ & $4(23,5)$ & \\
\hline \multicolumn{5}{|l|}{ Tasa de flujo salival estimulado } \\
\hline$<0.7 \mathrm{ml} / \mathrm{min}$ (disminuido) & $17(37,0)$ & $15(51,7)$ & $2(11,8)$ & \multirow{3}{*}{$0,017^{\prime}$} \\
\hline $0.7-1$ (normal) & $21(45,6)$ & $11(37,9)$ & $10(58,8)$ & \\
\hline$>1 \mathrm{ml} / \mathrm{min}$ (aumentado) & $8(17,4)$ & $3(10,3)$ & $5(29,4)$ & \\
\hline \multicolumn{5}{|c|}{ Aclaramiento de azúcar en saliva a los 15} \\
\hline minutos. & $39(84,8)$ & $24(82,8)$ & $15(88,2)$ & \multirow{2}{*}{$1,000^{\prime}$} \\
\hline $\begin{array}{l}\text { Coloracion azul } \\
\text { Coloración amarillenta }\end{array}$ & $7(15,2)$ & $5(17,2)$ & $2(11,8)$ & \\
\hline \multicolumn{5}{|c|}{ Prevalencia de caries dental y cálculo (IC 95\%) } \\
\hline Caries coronal (si/no) & $65,2(49,8-78,7)$ & $62,1(42,3-79,3)$ & $70,6(44,0-89,7)$ & 0,558 \\
\hline Caries radicular (si/no) & $34,8(21,4-50,3)$ & $31,0(15,3-50,8)$ & $41,9(18,4-67,1)$ & 0,486 \\
\hline Calculo dental (si/no) & $76,1(61,2-87,4)$ & $75,9(56,5-89,7)$ & $76,5(50,1-93,2)$ & 0,963 \\
\hline
\end{tabular}

* Test Exacto de Fisher ${ }^{*}$ Prueba $\mathrm{Chi}^{2}$ 
Al relacionar los factores asociados a la tasa de flujo salival no estimulado disminuida, se encontró una prevalencia menor de flujo salival disminuido en quienes se cepillaban los dientes tres o más veces al día (RP 0,42 IC 95\% 0,23 - 0,75) y presentaron cálculo localizado (RP 0,43 IC 95\% 0,30 - 0,85) comparado con quienes se cepillaban los dientes dos veces al día y no presentaron cálculos, respectivamente. Por otro lado, la prevalencia de flujo salival disminuido fue mayor en quienes usaban enjuague bucal ( $R P$ 1,87 IC 95\% 1,04 - 3,35) comparado con quienes no usaban enjuague. Ahora bien, la prevalencia de flujo salival estimulado disminuido fue menor en los participantes de sexo masculino comparado con los de sexo femenino (RP 0,19 IC 95\% 0,04 - 0,96) (Tabla 6).

Tabla 2. Factores sociodemográficos, antecedentes médicos, flujo salival y aclaramiento asociados a caries coronal.

\begin{tabular}{|c|c|c|c|c|c|c|}
\hline \multirow{2}{*}{ Variable } & \multirow{2}{*}{$\begin{array}{c}\text { Global } \\
\text { n (\%) }\end{array}$} & \multicolumn{2}{|c|}{ Caries Coronal } & \multirow{2}{*}{$\mathbf{R P}$} & \multirow{2}{*}{ IC $95 \%$} & \multirow{2}{*}{$\mathbf{p}$} \\
\hline & & Si & No & & & \\
\hline \multicolumn{7}{|l|}{ Nivel educativo } \\
\hline Analfabeta / Primaria & $16(34,8)$ & $10(62,5)$ & $6(37,5)$ & & & \\
\hline Secundaria & $18(39,1)$ & $12(66,7)$ & $6(33,3)$ & 1,07 & $0,64-1,76$ & 0,780 \\
\hline Estudios Superiores & $12(26,1)$ & $8(66,7)$ & $4(25,0)$ & 1,07 & $0,61-1,85$ & 0,820 \\
\hline \multicolumn{7}{|l|}{ Consumo de medicamentos } \\
\hline $\mathrm{Si}$ & $27(58,7)$ & $19(70,4)$ & $8(29,6)$ & 1,22 & $0,77-1,91$ & 0,382 \\
\hline No & $19(41,3)$ & $11(57,9)$ & $8(42,1)$ & & & \\
\hline \multicolumn{7}{|l|}{ Cepillado de dientes v/d } \\
\hline Dos & $14(30,4)$ & $12(85,7)$ & $2(14,3)$ & & & \\
\hline Tres o más & $32(69,6)$ & $18(56,2)$ & $14(43,8)$ & 0,66 & $0,45-0,95$ & 0,053 \\
\hline \multicolumn{7}{|l|}{ Frecuencia uso seda dental } \\
\hline Nunca & $16(34,8)$ & $10(62,5)$ & $6(37,5)$ & & & \\
\hline Semanal / Mensual & $12(26,1)$ & $8(66,7)$ & $4(33,3)$ & 1,07 & $0,61-1,85$ & 0,820 \\
\hline Diario & $18(39,1)$ & $12(66,7)$ & $6(37,5)$ & 1,07 & $0,65-1,76$ & 0,800 \\
\hline \multicolumn{7}{|l|}{ Uso de enjuague bucal } \\
\hline $\mathrm{Si}$ & $26(57,8)$ & $15(51,7)$ & $11(42,3)$ & 0,95 & $0,58-1,56$ & 0,832 \\
\hline No & $19(42,2)$ & $14(48,3)$ & $5(26,3)$ & & & \\
\hline \multicolumn{7}{|l|}{ Frec. de visita al odontólogo } \\
\hline Nunca & $4(8,7)$ & $4(13,3)$ & $0(0,0)$ & & & \\
\hline Una vez al año & $15(32,6)$ & $9(60,0)$ & $6(40,0)$ & 0,6 & $0,40-0,91$ & 0,126 \\
\hline Dos veces al año & $7(15,2)$ & $4(57,1)$ & $3(42,9)$ & 0,57 & $0,30-1,08$ & 0,125 \\
\hline Cuando duele & $20(43,5)$ & $13(65,0)$ & $7(35,0)$ & 0,65 & $0,47-0,89$ & 0,160 \\
\hline \multicolumn{7}{|l|}{ Valor de pH salival reportado } \\
\hline $5.7-7.6(\mathrm{n})$ & $20(43,5)$ & $12(60,0)$ & $8(40,0)$ & & & \\
\hline 7.6 ó más (básico) & $26(56,5)$ & $18(69,2)$ & $8(30,8)$ & 1,15 & $0,74-1,79$ & 0,515 \\
\hline \multicolumn{7}{|l|}{ Tasa flujo salival no estimulado } \\
\hline 0 - $0.25 \mathrm{ml} / \mathrm{min}$ (disminuido) & $27(58,7)$ & $19(70,4)$ & $8(29,6)$ & 1,14 & $0,70-1,88$ & 0,576 \\
\hline $0.25-0.35 \mathrm{ml} / \mathrm{min}$ (normal) & $13(28,3)$ & $8(61,5)$ & $5(38,5)$ & & & \\
\hline$>0.35 \mathrm{ml} / \mathrm{min}$ (aumentado) & $6(13,0)$ & $3(50,0)$ & $3(50,0)$ & 0,81 & $0,33-2,01$ & 0,636 \\
\hline \multicolumn{7}{|l|}{ Tasa de flujo salival estimulado } \\
\hline$<0.7 \mathrm{ml} / \mathrm{min}$ (disminuido) & $17(37,0)$ & $9(52,9)$ & $8(47,1)$ & 0,74 & $0,43-1,25$ & 0,240 \\
\hline $0.7-1 \mathrm{ml} / \mathrm{min}$ (normal) & $21(45,6)$ & $15(71,4)$ & $6(28,6)$ & & & \\
\hline$>1 \mathrm{ml}$ / min (aumentado) & $8(17,4)$ & $6(75,0)$ & $2(25,0)$ & 1,05 & $0,64-1,7$ & 0,858 \\
\hline \multicolumn{7}{|l|}{ Aclaramiento a 15 minutos. } \\
\hline Coloración amarillenta & $7(15,2)$ & $5(71,4)$ & $2(28,6)$ & 1,11 & $0,66-1,88$ & 0,708 \\
\hline
\end{tabular}


ARTÍCULO ORIGINAL

Tabla 3. Factores sociodemográficos, antecedentes médicos, flujo salival y aclaramiento asociados a caries radicular.

\begin{tabular}{|c|c|c|c|c|c|c|}
\hline \multirow{2}{*}{ Variable } & \multirow{2}{*}{$\begin{array}{c}\text { Global } \\
\text { n (\%) }\end{array}$} & \multicolumn{2}{|c|}{ Caries Radicular } & \multirow{2}{*}{$\mathbf{R P}$} & \multirow{2}{*}{ IC $95 \%$} & \multirow{2}{*}{$\mathbf{p}$} \\
\hline & & Si & No & & & \\
\hline \multicolumn{7}{|l|}{ Nivel educativo } \\
\hline Analfabeta / Primaria & $16(34,8)$ & $8(50,0)$ & $8(50,0)$ & & & \\
\hline Secundaria & $18(39,1)$ & $4(22,2)$ & $14(77,8)$ & 0,44 & $0,16-1,20$ & 0,091 \\
\hline Estudios Superiores & $12(26,1)$ & $4(33,3)$ & $8(66,7)$ & 0,67 & $0,26-1,70$ & 0,378 \\
\hline \multicolumn{7}{|l|}{ Consumo de medicamentos } \\
\hline $\mathrm{Si}$ & $27(58,7)$ & $10(37,0)$ & $17(63,0)$ & 1,17 & $0,51-2,68$ & 0,702 \\
\hline No & $19(41,3)$ & $6(31,6)$ & $13(68,4)$ & & & \\
\hline \multicolumn{7}{|l|}{ Cepillado de dientes v/d } \\
\hline Dos & $14(30,4)$ & $3(21,4)$ & $11(78,6)$ & & & \\
\hline Tres o más & $32(69,6)$ & $13(40,6)$ & $19(59,4)$ & 1,89 & $0,63-5,62$ & 0,209 \\
\hline \multicolumn{7}{|l|}{ Frecuencia uso seda dental } \\
\hline Nunca & $16(34,8)$ & $9(56,3)$ & $7(43,7)$ & & & \\
\hline Semanal / Mensual & $12(26,1)$ & $3(25,0)$ & $9(75,0)$ & 0,44 & $0,15-1,29$ & 0,982 \\
\hline Diario & $18(39,1)$ & $4(22,2)$ & $14(77,8)$ & 0,39 & $0,15-1,04$ & 0,076 \\
\hline \multicolumn{7}{|l|}{ Uso de enjuague bucal } \\
\hline $\mathrm{Si}$ & $26(57,8)$ & $7(26,9)$ & $19(73,1)$ & 0,64 & $0,28-1,45$ & 0,286 \\
\hline No & $19(42,2)$ & $8(42,1)$ & $11(57,9)$ & & & \\
\hline \multicolumn{7}{|l|}{ Frec. de visita al odontólogo } \\
\hline Nunca & $4(8,7)$ & $4(13,3)$ & $0(0,0)$ & & & \\
\hline Una vez al año & $15(32,6)$ & $4(26,7)$ & $11(73,3)$ & 0,26 & $0,12-0,62$ & 0,008 \\
\hline Dos veces al año & $7(15,2)$ & $0(0,0)$ & $7(100,0)$ & - & - & - \\
\hline Cuando duele & $20(43,5)$ & $8(40,0)$ & $12(60,0)$ & 0,40 & $0,23-0,68$ & 0,028 \\
\hline \multicolumn{7}{|l|}{ Valor de $\mathrm{pH}$ salival reportado } \\
\hline $5.7-7.6(\mathrm{n})$ & $20(43,5)$ & $7(35,0)$ & $13(65,0)$ & & & \\
\hline 7.6 ó más (básico) & $26(56,5)$ & $9(34,6)$ & $17(65,4)$ & 0,98 & $0,45-2,19$ & 0,978 \\
\hline \multicolumn{7}{|l|}{ Tasa flujo salival no estimulado } \\
\hline $0-0.25 \mathrm{ml} / \mathrm{min}$ (disminuido) & $27(58,7)$ & $8(29,6)$ & $19(70,4)$ & 0,77 & $0,31-1,89$ & 0,576 \\
\hline $0.25-0.35 \mathrm{ml} / \mathrm{min}$ (normal) & $13(28,3)$ & $5(38,5)$ & $8(61,5)$ & & & \\
\hline$>0.35 \mathrm{ml} / \mathrm{min}$ (aumentado) & $6(13,0)$ & $3(50,0)$ & $3(50,0)$ & 1,3 & $0,45-3,73$ & 0,636 \\
\hline \multicolumn{7}{|l|}{ Tasa de flujo salival estimulado } \\
\hline$<0.7 \mathrm{ml} / \mathrm{min}$ (disminuido) & $17(37,0)$ & $7(41,2)$ & $10(58,8)$ & 2,8 & $0,87-9,49$ & 0,061 \\
\hline $0.7-1 \mathrm{ml} / \mathrm{min}$ (normal) & $21(45,6)$ & $3(14,3)$ & $18(85,7)$ & & & \\
\hline$>1 \mathrm{ml} /$ min (aumentado) & $8(17,4)$ & $6(75,0)$ & $2(25,0)$ & 5,3 & $1,7116,11$ & 0,002 \\
\hline \multicolumn{7}{|l|}{ Aclaramiento a 15 minutos. } \\
\hline Coloración azul & $39(84,8)$ & $13(33,3)$ & $26(66,7)$ & & & \\
\hline Coloración amarillenta & $7(15,2)$ & $3(42,9)$ & $4(57,1)$ & 1,28 & $0,49-3,37$ & 0,626 \\
\hline
\end{tabular}


Tabla 4. Factores sociodemográficos, antecedentes médicos, flujo salival y aclaramiento asociados a cálculo dental.

\begin{tabular}{|c|c|c|c|c|c|c|}
\hline \multirow{2}{*}{ Variable } & \multirow{2}{*}{$\begin{array}{c}\text { Global } \\
\text { n (\%) }\end{array}$} & \multicolumn{2}{|c|}{ Cálculo Dental } & \multirow{2}{*}{$\mathbf{R P}$} & \multirow{2}{*}{ IC $95 \%$} & \multirow{2}{*}{$\mathrm{p}$} \\
\hline & & Si & No & & & \\
\hline \multicolumn{7}{|l|}{ Nivel educativo } \\
\hline Analfabeta / Primaria & $16(34,8)$ & $14(87,5)$ & $2(12,5)$ & & & \\
\hline Secundaria & $18(39,1)$ & $13(72,2)$ & $5(27,8)$ & 0,83 & $0,59-1,16$ & 0,272 \\
\hline Estudios Superiores & $12(26,1)$ & $8(66,7)$ & $4(33,3)$ & 0,76 & $0,49-1,18$ & 0,184 \\
\hline \multicolumn{7}{|l|}{ Consumo de medicamentos } \\
\hline $\mathrm{Si}$ & $27(58,7)$ & $22(81,5)$ & $5(18,5)$ & 1,19 & $0,84-1,70$ & 0,307 \\
\hline No & $19(41,3)$ & $13(68,4)$ & $6(31,6)$ & & & \\
\hline \multicolumn{7}{|l|}{ Cepillado de dientes v/d } \\
\hline Dos & $14(30,4)$ & $13(92,9)$ & $1(7,1)$ & & & \\
\hline Tres o más & $32(69,6)$ & $13(68,8)$ & $10(31,3)$ & 0,74 & $0,56-0,97$ & 0,078 \\
\hline \multicolumn{7}{|l|}{ Frecuencia uso seda dental } \\
\hline Nunca & $16(34,8)$ & $14(87,5)$ & $2(12,5)$ & & & \\
\hline Semanal / Mensual & $12(26,1)$ & $10(83,3)$ & $2(16,7)$ & 0,95 & $0,70-1,30$ & 1,000 \\
\hline Diario & $18(39,1)$ & $11(61,1)$ & $7(38,9)$ & 0,70 & $0,46-1,05$ & 0,125 \\
\hline \multicolumn{7}{|l|}{ Uso de enjuague bucal } \\
\hline $\mathrm{Si}$ & $26(57,8)$ & $20(76,9)$ & $6(23,1)$ & & & \\
\hline No & $19(42,2)$ & $14(73,7)$ & $11(57,9)$ & 1,04 & $0,74-1,47$ & 0,825 \\
\hline \multicolumn{7}{|l|}{ Frec. de visita al odontólogo } \\
\hline Nunca & $4(8,7)$ & $3(75,0)$ & $1(25,0)$ & & & \\
\hline Una vez al año & $15(32,6)$ & $13(86,7)$ & $2(13,3)$ & 1,15 & $0,63-2,10$ & 0,570 \\
\hline Dos veces al año & $7(15,2)$ & $4(57,1)$ & $3(42,9)$ & 0,76 & $0,32-1,79$ & 0,554 \\
\hline Cuando duele & $20(43,5)$ & $15(75,0)$ & $5(25,0)$ & 1,00 & $0,53-1,86$ & 1,000 \\
\hline \multicolumn{7}{|l|}{ Valor de pH salival reportado } \\
\hline 5.7- $7.6(\mathrm{n})$ & $20(43,5)$ & $16(80,0)$ & $4(20,0)$ & & & \\
\hline 7.6 ó más (básico) & $26(56,5)$ & $19(73,1)$ & $7(26,9)$ & 0,91 & $0,66-1,26$ & 0,585 \\
\hline \multicolumn{7}{|l|}{ Tasa flujo salival no estimulado } \\
\hline $0-0.25 \mathrm{ml} / \mathrm{min}$ (disminuido) & $27(58,7)$ & $18(66,7)$ & $9(33,3)$ & 0,79 & $0,55-1,12$ & 0,234 \\
\hline $0.25-0.35 \mathrm{ml} / \mathrm{min}$ (normal) & $13(28,3)$ & $11(84,6)$ & $2(15,4)$ & & & \\
\hline$>0.35 \mathrm{ml} / \mathrm{min}$ (aumentado) & $6(13,0)$ & $6(100,0)$ & $0(0,0)$ & 1,18 & $0,94-1,49$ & 0,310 \\
\hline \multicolumn{7}{|l|}{ Tasa de flujo salival estimulado } \\
\hline$<0.7 \mathrm{ml} / \mathrm{min}$ (disminuido) & $17(37,0)$ & $12(70,6)$ & $5(29,4)$ & 0,99 & $0,65-1,49$ & 0,955 \\
\hline $0.7-1 \mathrm{ml} / \mathrm{min}$ (normal) & $21(45,6)$ & $15(71,4)$ & $6(28,6)$ & & & \\
\hline$>1 \mathrm{ml} / \min$ (aumentado) & $8(17,4)$ & $8(100,0)$ & $0(0,0)$ & 1,4 & $1,07-1,83$ & 0,090 \\
\hline \multicolumn{7}{|l|}{ Aclaramiento a 15 minutos. } \\
\hline Coloración azul & $39(84,8)$ & $30(76,9)$ & $9(23,1)$ & & & \\
\hline Coloración amarillenta & $7(15,2)$ & $5(71,4)$ & $2(28,6)$ & 0,93 & $0,56-1,53$ & 0,754 \\
\hline
\end{tabular}


ARTÍCULO ORIGINAL

Tabla 5. Factores sociodemográficos, antecedentes médicos, higiene oral, flujo y aclaramiento asociados a pH básico.

\begin{tabular}{|c|c|c|c|c|c|c|}
\hline \multirow{2}{*}{ Variables } & \multirow{2}{*}{$\begin{array}{c}\text { Global } \\
\text { n (\%) }\end{array}$} & \multicolumn{2}{|c|}{ pH salival } & \multirow{2}{*}{$\mathbf{R P}$} & \multirow{2}{*}{ IC 95\% } & \multirow[b]{2}{*}{$\mathbf{P}$} \\
\hline & & Básico & Neutro & & & \\
\hline \multicolumn{7}{|c|}{ Características sociodemográficas } \\
\hline \multicolumn{7}{|c|}{ Sexo } \\
\hline Femenino & $29(63,0)$ & $17(58,6)$ & $12(41,4)$ & & & \\
\hline Masculino & $17(37,0)$ & $9(52,9)$ & $8(47,1)$ & 0,90 & $0,52-1,55$ & 0,708 \\
\hline \multicolumn{7}{|l|}{ Edad } \\
\hline $50-55$ & $20(43,5)$ & $12(60,0)$ & $8(40,0)$ & & & \\
\hline$\geq 56$ & $26(56,5)$ & $14(53,9)$ & $12(46,2)$ & 0,89 & $0,54-1,48$ & 0,676 \\
\hline \multicolumn{7}{|l|}{ Actualmente fuma } \\
\hline $\mathrm{Si}$ & $7(15,2)$ & $2(28,6)$ & $5(71,4)$ & \multirow[t]{2}{*}{0,46} & \multirow[t]{2}{*}{$0,14-1,54$} & \multirow[t]{2}{*}{0,105} \\
\hline No & $39(84,8)$ & $24(61,5)$ & $15(38,5)$ & & & \\
\hline \multicolumn{7}{|l|}{ Padece de alguna enfermedad } \\
\hline $\mathrm{Si}$ & $31(67,4)$ & $17(54,8)$ & $14(15,2)$ & \multirow[t]{2}{*}{0,91} & \multirow[t]{2}{*}{$0,54-1,54$} & \multirow[t]{2}{*}{0,741} \\
\hline No & $15(32,6)$ & $9(60,0)$ & $6(40,0)$ & & & \\
\hline \multicolumn{7}{|l|}{ Consumo de medicamentos } \\
\hline $\mathrm{Si}$ & $26(56,5)$ & $15(57,7)$ & $11(42,3)$ & \multirow{2}{*}{1,05} & \multirow{2}{*}{$0,63-1,76$} & 0.855 \\
\hline No & $20(43,5)$ & $11(55,0)$ & $9(45,0)$ & & & \\
\hline $\begin{array}{l}\text { Hábitos de higiene oral y cond } \\
\text { Número de veces al día de cep } \\
\text { dientes }\end{array}$ & & & & & & \\
\hline Dos & $14(30,4)$ & $10(71,4)$ & $4(28,6)$ & & & \\
\hline Tres o más & $32(69,6)$ & $16(50,0)$ & $16(20,0)$ & 0,7 & $0,43-1,13$ & 0,177 \\
\hline Frecuencia de uso de la seda d & & & & & & \\
\hline Nunca & $16(34,8)$ & $6(37,5)$ & $10(62,5)$ & & & \\
\hline Semanal/Mensual & $12(26,1)$ & $7(58,3)$ & $5(41,7)$ & 1,55 & $0,70-3,44$ & 0,274 \\
\hline Diario & $18(39,1)$ & $13(72,2)$ & $5(27,8)$ & 1,92 & $0,96-3,86$ & 0,082 \\
\hline Uso de enjuague bucal & & & & & & \\
\hline $\mathrm{Si}$ & $26(57,7)$ & $14(53,8)$ & $12(46,1)$ & 0,85 & $0,52-1,30$ & 0,532 \\
\hline $\begin{array}{l}\text { No } \\
\text { Frecuencia de visita al odontó }\end{array}$ & $19(42,2)$ & $12(63,2)$ & $7(36,8)$ & & & \\
\hline Nunca & $4(8,7)$ & $1(25,0)$ & $3(75,0)$ & & & \\
\hline Una vez al año & $15(32,6)$ & $9(60,0)$ & $6(40,0)$ & 2,4 & $0,42-13,77$ & 0,213 \\
\hline Dos veces al año & $7(15,2)$ & $3(42,9)$ & $4(57,1)$ & 1,7 & $0,26-11,47$ & 0,554 \\
\hline Cuando duele & $20(43,5)$ & $13(65,0)$ & $7(35,0)$ & 2,6 & $0,46-14,63$ & 0,138 \\
\hline Presencia de prótesis dental & & & & & & \\
\hline $\mathrm{Si}$ & $34(73,9)$ & $20(58,8)$ & $14(41,2)$ & 1,2 & $0,62-2,21$ & 0,596 \\
\hline No & $12(26,1)$ & $6(50,0)$ & $6(50,0)$ & & & \\
\hline Presencia de dientes obturado & & & & & & \\
\hline $\mathrm{Si}$ & $41(89,1)$ & $25(60,0)$ & $16(39,0)$ & 3,05 & $0,52-17,90$ & 0,081 \\
\hline $\begin{array}{l}\text { No } \\
\text { Presencia de cálculo dental }\end{array}$ & $5(10,87)$ & $1(20,0)$ & $4(80,0)$ & & & \\
\hline $\mathrm{Si}$ & $35(76,1)$ & $19(54,3)$ & $16(45,7)$ & 0,85 & $0,40-1,46$ & 0,585 \\
\hline No & $11(23,9)$ & $7(63,6)$ & $4(36,4)$ & & & \\
\hline Propiedades de la saliva & & & & & & \\
\hline Tasa de flujo salival no estimu & & & & & & \\
\hline $0-0.25 \mathrm{ml} / \mathrm{min}$ (disminuido) & $27(58,7)$ & $14(51,9)$ & $13(48,1)$ & 0,96 & $0,52-1,79$ & 0,906 \\
\hline $0.25-0.35 \mathrm{ml} / \mathrm{min}$ (normal). & $13(28,3)$ & $7(53,9)$ & $6(46,1)$ & & & \\
\hline$>0.35 \mathrm{ml} / \mathrm{min}$. (aumentado) & $6(13,0)$ & $5(83,3)$ & $1(16,7)$ & 1,54 & $0,83-2,87$ & 0,216 \\
\hline Tasa de flujo salival estimulad & & & & & & \\
\hline$<0.7 \mathrm{ml} / \mathrm{min}$ (disminuido) & $17(37,0)$ & $8(47,1)$ & $9(52,9)$ & 0,82 & $0,44-1,54$ & 0,536 \\
\hline $0.7-1$ (normal). & $21(45,6)$ & $12(57,1)$ & $9(42,9)$ & & & \\
\hline >1ml/min (aumentado) & $8(17,4)$ & $6(75,0)$ & $2(25,0)$ & 1,31 & $0,76-2,26$ & 0,376 \\
\hline Aclaramiento a los 15 minutos & & & & & & \\
\hline Coloración azul & $39(84,8)$ & $23(59,0)$ & $16(41,0)$ & & & \\
\hline Coloración amarillenta & $7(15,2)$ & $3(42,9)$ & $4(57,1)$ & 0,73 & $0,30-1,78$ & 0,428 \\
\hline
\end{tabular}

50 
Tabla 6. Factores asociados a la tasa de flujo salival no estimulado y estimulado disminuida.

\begin{tabular}{|c|c|c|c|c|c|c|}
\hline \multirow[t]{2}{*}{ Variable } & \multicolumn{3}{|c|}{$\begin{array}{l}\text { Tasa de flujo salival no } \\
\text { estimulado disminuida }\end{array}$} & \multicolumn{3}{|c|}{$\begin{array}{c}\text { Tasa de flujo salival estimulado } \\
\text { disminuida }\end{array}$} \\
\hline & $\mathbf{R P}$ & IC $95 \%$ & $\mathbf{p}$ & $\mathbf{R P}$ & IC $95 \%$ & $\mathbf{p}$ \\
\hline Sexo masculino & 1,16 & $0,46-2,91$ & 0,748 & 0,19 & $0,04-0,96$ & 0,045 \\
\hline Edad $\geq 56$ & 1,45 & $0,81-2,59$ & 0,211 & 2,21 & $0,66-7,43$ & 0,200 \\
\hline Estrato socioeconómico 3 y 4 & 1,74 & $0,60-5,05$ & 0,308 & 0,36 & $0,17-1,13$ & 0,081 \\
\hline Fumador actual & 1,07 & $0,40-2,87$ & 0,892 & 1,17 & $0,27-4,96$ & 0,833 \\
\hline Antiglicémico & 0,96 & $0,32-2,87$ & 0,939 & 1,16 & $0,20-6,52$ & 0,869 \\
\hline Otros medicamentos & 1,10 & $0,43-2,78$ & 0,843 & 1,48 & $0,21-10,14$ & 0,691 \\
\hline No aplica medicamento & 0,89 & $0,44-1,78$ & 0,737 & 0,84 & $0,18-3,86$ & 0,821 \\
\hline Cepilla dientes 3 o más veces/día & 0,42 & $0,23-0,75$ & 0,004 & 0,68 & $0,20-2,33$ & 0,542 \\
\hline Uso de seda dental semanal/mensual & 0,72 & $0,29-1,82$ & 0,492 & 0,47 & $0,13-1,70$ & 0,250 \\
\hline Uso de seda dental diario & 0,73 & $0,32-1,65$ & 0,447 & 0,78 & $0,28-2,13$ & 0,626 \\
\hline Uso de enjuague bucal & 1,87 & $1,04-3,35$ & 0,036 & 1,46 & $0,48-4,38$ & 0,502 \\
\hline Visita al odontólogo 1 ó 2 veces/año & 1,09 & $0,69-1,93$ & 0,761 & 1,00 & $0,49-2,24$ & 0,993 \\
\hline Nunca visita al odontólogo & 1,94 & $0,69-5,47$ & 0,211 & 1,09 & $0,19-6,30$ & 0,922 \\
\hline Uso de prótesis & 0,69 & $0,39-1,29$ & 0,206 & 1,50 & $0,37-6,04$ & 0,566 \\
\hline Calculo localizado & 0,43 & $0,30-0,85$ & 0,015 & 0,52 & $0,15-1,83$ & 0,311 \\
\hline Calculo generalizado & 0,53 & $0,23-1,26$ & 0,150 & 0,47 & $0,11-1,97$ & 0,300 \\
\hline pH salival básico & 1,05 & $0,46-2,39$ & 0,914 & 0,62 & $0,29-1,82$ & 0,383 \\
\hline $\begin{array}{l}\text { Aclaramiento de azúcar en saliva } \\
\text { coloración amarillenta }\end{array}$ & 1,23 & $0,53-2,90$ & 0,627 & 0,78 & $0,30-2,07$ & 0,616 \\
\hline
\end{tabular}

\section{DISCUSIÓN}

Se encontró una prevalencia de caries coronal y radicular del $65,2 \%$ y del $34,8 \%$ respectivamente; cifras inferiores a las reportadas por Castrejón y colaboradores en pacientes mayores de 60 años quienes reportaron caries coronal de $90,8 \%$ y de caries radicular de $46,9 \%$, inferiores también a reportes de México dados por la Organización Mundial de la Salud con más del $90 \%$ de caries dental de la población. ${ }^{13}$ Esto indica la necesidad de buscar estrategias que incluyan medidas efectivas para la prevención y el control de la caries dental en pacientes adultos mayores

La prevalencia de cálculo dental encontrada fue de $76,1 \%$ cifra elevada al compararla con el estudio de Castrejón y colaboradores, quienes encontraron que el $41,8 \%$ presentaba una zona dental o más cubierta por cálculo en al menos dos terceras partes. ${ }^{13}$ Adicionalmente, se ha confirmado que un flujo salival elevado proporciona un aumento en el $\mathrm{pH}$, lo que ocasiona mineralización de la placa bacteriana, formación de cálculo dental y presencia de enfermedad periodontal. ${ }^{6} \mathrm{El}$ presente estudio corrobora que el aumento del flujo salival tanto estimulado como no estimulado es factor de riesgo para cálculo dental (RP 1,4 IC 95\% 1,07 - 1,83), aunque estas cifras no son estadísticamente significativas.

En cuanto al valor del pH salival, se observó que fue normal en el $43,5 \%$ y básico en el $56,5 \%$ de los participantes. Así mismo, se encontró una razón de prevalencia de 1,05, indicando que el consumo de medicamentos es un posible factor de riesgo para el aumento del pH salival, pero no fue estadísticamente significativa esta asociación. En relación con el aclaramiento salival mayor a los 15 minutos en el $84.8 \%$ y los demás datos mencionados cabe destacar que efectivamente al ser la saliva un factor importante en el mantenimiento de equilibrio en el proceso de mineralización y desmineralización. ${ }^{14}$ Los cambios de $\mathrm{pH}$ ocasionan un ambiente favorable a los mecanismos de formación de cálculo y erosión del esmalte favorecidos además por el tiempo de permanencia de los alimentos en la cavidad bucal. 
El sexo femenino presentó una tasa de flujo salival estimulado disminuida $(51,7 \%)$ comparado con los hombres $(11,8 \%)$, con un comportamiento estadísticamente significativo $(\mathrm{p}=0,017)$. De igual manera, los resultados obtenidos por Pajukoski y colaboradores en un estudio con 169 pacientes, mostraron que la tasa de flujo salival estimulado para los hombres estuvo entre 0.9 y $0.7 \mathrm{ml} / \mathrm{min}$ en el $48 \%$ de los participantes y para mujeres entre 0.6 y $0.6 \mathrm{mil} / \mathrm{min}$ en el $62.5 \%$ de los participantes $(\mathrm{p}<0.05)$, igualmente estos resultados son estadísticamente significativos. ${ }^{2}$ En contraste, Sáez y colabodores (2007), reportaron que en las mujeres la reducción del flujo salival se acentúa después de la menopausia más exactamente después de los 60 años..$^{15}$ Estos resultados fueron similares a los obtenidos por Parvinen y Larmas, quienes realizaron un estudio en 117 mujeres finlandesas mayores de 50 años de edad posmenopáusica; ${ }^{16}$ no obstante estas diferencias no fueron significativas. Cabe la preocupación de modificar hábitos para elevar la tasa de flujo de saliva en mujeres en edades mayores de 40 años ya que al parecer los cambios hormonales se relacionan con bajas en la cantidad de saliva secretada y se ha demostrado la relación directa entre flujo disminuido y caries dental.

El análisis del flujo salival mostró relación entre la tasa de flujo salival estimulado aumentada y disminuida con caries radicular; en este sentido, el flujo salival normal es protector para la presencia de caries radicular. Efectos similares se encontraron en el estudio de Stookey, cuyos resultados mostraron que una deficiente secreción salival así como una saliva espesa y viscosa se constituyen en factor predisponente al aumentar la tendencia de caries dental. ${ }^{17}$ Esta relación también fue estudiada por Jiménez en mujeres gestantes, quien concluyó que una alteración en la composición de la saliva, al igual que la disminución del $\mathrm{pH}$ o flujo salival y/o un aumento de la viscosidad salival pueden experimentar un índice más alto de caries dental. ${ }^{18}$

Lo anterior se explica en parte, porque el flujo salival disminuido proporciona bajas de $\mathrm{pH}$ salival y permite un medio ecológico ideal para las bacterias cariogénicas, ${ }^{1,6,19}$ a su vez, a menor tasa de flujo menor capacidad de aclaramiento lo cual conlleva aumento en la aparición de lesiones cariosas. ${ }^{7}$ De igual manera, la reducción salival puede deberse a diversos factores como la ingesta de medicamentos y alteraciones sistémicas crónicas..$^{10}$ Estos datos confirman nuevamente los concejos de investigadores de buscar medidas que estimulen el flujo salival como estrategia para disminuir la prevalencia de enfermedades bucales. ${ }^{14}$

\section{Conclusiones}

Presentar una tasa de flujo salival estimulado aumentado aumenta el riesgo de cálculo dental.

- La tasa de flujo salival estimulado disminuida prevalece más en mujeres que en hombres.

- Tener 56 años o más, se constituye en un factor de riesgo en cuanto a la disminución de flujo salival estimulado aumenta la prevalencia de presentar flujo salival estimulado disminuido.

- Hay mayor prevalencia de $\mathrm{pH}$ básico en personas no fumadoras.

- El flujo salival aumentado es factor de riesgo para tener cálculo dental.

- El sexo masculino está ligado con la presencia de caries coronal y radicular.

- Se debe buscar estrategias para estimular el flujo salival para disminuir la prevalencia de enfermedades bucales.

\section{BIBLIOGRAFÍA}

1. Bardow A, Ten Cate JM, Nauntofte B, Nyvad B. Effect of unstimulated saliva flow rate on experimental root caries. Caries Res 2003; 37: 232 - 236.

2. Pajukoski H, Meurman JH, Snellman-Gröhn S, Keinänen, S. Sulkava, R. Salivary flow and composition in elderly patients referred to an acute care geriatric ward. Oral Surg Oral Med Oral Pathol Oral Radiol Endod 1997; 84: $265-271$.

3. Fischer, D. Ship JA. Effect of age on variability of parotid salivary gland flow rates over time. Age Ageing 1999; 28 : $557-561$.

4. López SM, Ochoa LF, Valle NM, Aránzazu GC. Flujo salival en adultos mayores y su relación con enfermedades crónicas y el consumo de medicamentos. Ustasalud 2007; 6: 9 - 16.

5. Chimenos K, Márquez S. Burning mouth and saliva. Medicina Oral 2002; 7: 244 - 253.

6. Walsh L. Aspectos clínicos de biología salival para el clínico dental. Revista de Mínima Intervención en Odontología [en línea]. 2008 [fecha de acceso: 3 de noviembre de 2009]; 1 (1): [aprox. 18 p.]. URL disponible en: http:// www.midentistry.com/JMID-1S-1.pdf.

7. Loyo K, Balda R, Gonzalez O, Solorzano A, Gonzalez M. Actividad cariogénica y su relación con flujo salival y la capacidad amortiguadora de la saliva. Acta Odontológica Venez [en línea] 1999 [fecha de acceso: 19 de septiembre de 2009]; 37. URL disponible en: http://www.scielo.org.ve/ scielo.php?pid $=$ S0001-3651999000300003\&script $=$ sci arttext.

8. Henostroza, G. Márquez, F. Webb, J. Exámenes complementarios para determinar el riesgo de caries. En: Henostroza G. Caries Dental, Principios y Procedimientos para el Diagnóstico. Perú: Universidad Peruana Cayetano Heredia; 2007. p. 89 - 104.

9. Thylstrup A, Fejerskov O. Caries. Ediciones Doyma S.A; 1988. p. 16- 18, 24, 124-125.

10. Ikebe K, Matsuda K, Morrii K, Hazeyama T, Kagawa R, Ogawa T, Nokubi T. Relationship between bite force and salivary flow in older adults. Oral Surg Oral Med Oral Pa- 


\section{ARTÍCULO ORIGINAL}

thol Oral Radiol Endod 2007; 104: 510 - 515.

11. Almstahl A, Wikström M, Stemberg I, Jakobsson A, Fagerberg-Mohlin B. Oral microbiota associated with hyposalivation of different origins. Oral Micriobio Immunol 2003; 18: $1-8$.

12. Goulet D, Brudevold F, Tehrani A, Attarzadeh F. Sugar clearance from saliva and intra-oral spaces. J Dent Res 1985; 64: 411 - 415.

13. Castrejón RC, Borges SA, Irigoyen ME. Validación de un instrumento para medir el efecto de la salud bucal en la calidad de vida de adultos mayores mexicanos. Rev Panam Salud 2010; 27: 321 - 329.

14. Ortega ME, Calzado M, Márquez M. Evaluación del flujo y viscosidad salival y su relación con el índice de caries. Instituto Superior de Ciencias Médicas MEDISAN 1998; 2: $33-39$.

15. Sáez R, Carmona M, Jiménez Z, Alfaro X. Cambios bucales en el adulto mayor. Rev Cubana Estomatol [en línea] 2007 [fecha de acceso: 5 de marzo de 2010]; 44 (4). URL disponible en: http://scielo.sld.cu/scielo.php?script = sci arttext\&pid $=$ S0034-75072007000400011\&lng = en

16. Parvinen T, Larmas M. Age dependency of simulated salivary flow rate, $\mathrm{pH}$, and lactobacillus and yeast concentrations. J Dent Res 1982; 61: 1052.

17. Stookey GK. The effect of saliva on dental caries. J Am Dent Assoc 2008; 139: 1s - 17s.

18. Jiménez RI. Importancia del ph, flujo y viscosidad salival sobre el desarrollo de caries dental en mujeres gestantes del primer trimestre [Tesis Doctoral]. Lima, Perú: Universidad Nacional Mayor de San Marcos, 2004. URL disponible en: http//:www.cybertesis.edu.pe/sisbib/2004/jimenez_mr/html/index-frames.html

19. García R, Ávila A. Zaragoza M. Cruz V. Moreno A. Asociación entre microorganismos y la capacidad amortiguadora de la saliva con la caries dental de escolares. Revista Odontológica Mexicana [en línea] 2008 [fecha de acceso: 18 de septiembre de 2009]; 12 (4): URL disponible en: http://www. journals. unam.mx/index.php/rom/article/view/15624.

Correos electrónicos de los autores:

Carmen Alodia Martínez López: carmenmartinez82@yahoo.com, Tatiana Marcela Agudelo Osorio: tatianaagudelo184@hotmail.com, Ana Lucía Espejo Pérez: pili 996@hotmail.com,

Zareth Garzón Ramírez: zareth121@hotmail.com,

Katherine Martínez Maldonado: misskatys27@hotmail.com

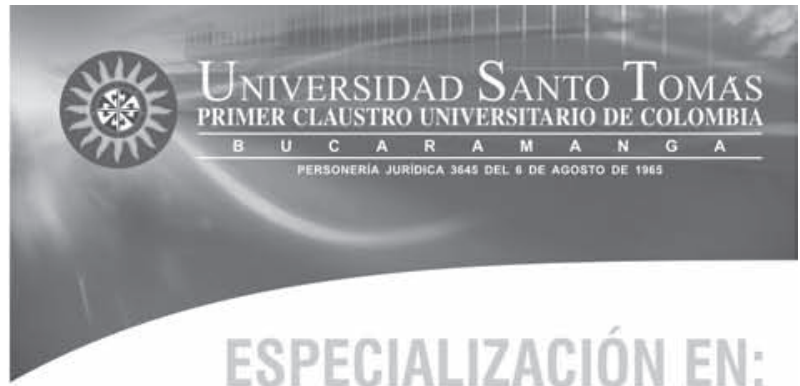

ENDODONCIA

SNIES 4907 ODONTOPEDIATRÍA

SNIES 9535

ORTODONCIA

SNIES 9537

REHABILITACIÓN ORAL

SNIES 9636

\author{
www.ustabuca.edu.co \\ e-mail: mercadeo@mail.ustabuca.edu.co
}

6800801 Ext. 1801 - 1263 - 1264 - Linea Gratuita 018000917044

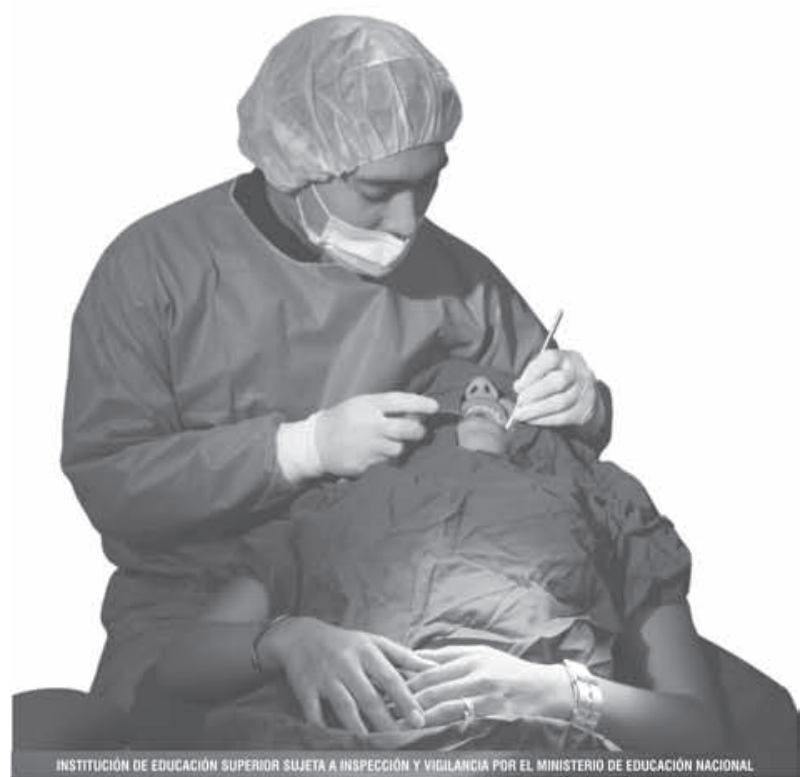

